\title{
National roll-out of latent tuberculosis testing and treatment for new migrants in England: a retrospective evaluation in a high-incidence area
}

\author{
Miranda G. Loutet ${ }^{1}$, Matthew Burman ${ }^{2,3}$, Nivenka Jayasekera ${ }^{3}$, \\ Duncan Trathen ${ }^{4}$, Susan Dart ${ }^{3}$, Heinke Kunst ${ }^{2,3}$ and Dominik Zenner ${ }^{1,5,6}$
}

Affiliations: ${ }^{1}$ National Infection Service, Public Health England, London, UK. ${ }^{2}$ Blizard Institute, Barts and The London School of Medicine and Dentistry, Queen Mary University, London, UK. ${ }^{3}$ Dept of Respiratory Medicine, Barts Health NHS Trust, London, UK. ${ }^{4}$ Newham Clinical Commissioning Group, London, UK. ${ }^{5}$ Institute for Global Health, University College London, London, UK. ${ }^{6}$ National Institute for Health Research Health Protection Research Unit in Respiratory Infections, Imperial College London, London, UK.

Correspondence: Miranda G. Loutet, Public Health England, 61 Colindale Ave, London, NW9 5EQ, UK. E-mail: mirandagloutetagmail.com

@ERSpublications

The LTBI screening programme is effective in identifying a high number of LTBI cases, but it is patient and provider-dependent http://ow.ly/AKMu30g1rMB

Cite this article as: Loutet $\mathrm{MG}$, Burman $\mathrm{M}$, Jayasekera $\mathrm{N}$, et al. National roll-out of latent tuberculosis testing and treatment for new migrants in England: a retrospective evaluation in a high-incidence area. Eur Respir J 2018; 51: 1701226 [https://doi.org/10.1183/13993003.01226-2017].

ABSTRACT Latent tuberculosis infection (LTBI) screening is an important intervention for tuberculosis (TB) elimination in low-incidence countries and is, therefore, a key component of England's TB control strategy. This study describes outcomes from a LTBI screening programme in a high-incidence area to inform national LTBI screening in England and other low-incidence countries.

We conducted a retrospective cohort study of LTBI screening among eligible migrants (from highincidence countries and entered the UK within the last 5 years), who were identified at primary-care clinics in Newham, London between August 2014 and August 2015. Multivariable logistic regression was used to identify factors associated with LTBI testing uptake, interferon- $\gamma$ release assay (IGRA) positivity and treatment uptake.

$40 \%$ of individuals offered LTBI screening received an IGRA test. The majority of individuals tested were 16-35 years old, male and born in India, Bangladesh or Pakistan. Country of birth, smoking status and co-morbidities were associated with LTBI testing uptake. IGRA positivity was $32 \%$ among those tested and was significantly associated with country of birth, age, sex and co-morbidities.

This study identifies factors associated with screening uptake, IGRA positivity and treatment uptake, and improves understanding of groups that should be supported to increase acceptability of LTBI testing and treatment in the community.

This article has supplementary material available from erj.ersjournals.com

Received: June 202017 | Accepted after revision: Oct 062017

Ethics approval: Public Health England has authority under the NHS Act 2012 to hold and analyse national screening data from the LTBI testing and treatment programme for public health, monitoring and evaluation purposes.

Conflict of interest: None declared.

Copyright $\odot$ ERS 2018. This ERJ Open article is open access and distributed under the terms of the Creative Commons Attribution Non-commercial Licence 4.0. 


\section{Introduction}

The World Health Organization's "End TB Strategy" aims to significantly reduce global tuberculosis (TB) incidence and mortality [1]. The strategy also aims for TB elimination (defined as incidence of $<1$ per 1000000 people) in low-incidence countries. One of the main interventions to achieve these goals is latent TB infection (LTBI) screening of high-risk groups, including migrants, to prevent re-activation [2].

In 2014, there were 6520 cases of TB notified in England, a rate of 12.0 cases per 100000 [3]. Nearly three-quarters $(72.2 \%)$ of all $\mathrm{TB}$ cases were notified among the foreign-born population and the vast majority $(86.0 \%)$ of foreign-born cases were notified among settled migrants who had been in the UK for more than 2 years [3]. It is likely that the overwhelming majority of $\mathrm{TB}$ cases in the foreign-born population are due to reactivation of LTBI acquired before arrival in the UK, a pattern which has been observed in other countries [3-5].

It is well known that treatment for LTBI is efficacious in preventing active TB $[6,7]$. Studies in the UK have also shown that targeted programmes using primary-care registers [8] and interferon- $\gamma$ release assays (IGRA) to screen those most at risk of developing active TB are more effective in identifying LTBI cases, preventing active TB and are cost effective $[9,10]$. Based on this evidence, new entrant LTBI testing and treatment became a key intervention of the Collaborative TB Strategy for England 2015-2020, which aims to reduce TB incidence in England and reduce health inequalities from TB over the next 5 years [11]. Systematic national implementation of the LTBI programme is essential to achieving the aims of the collaborative strategy and supports the WHO goal of TB elimination.

In 2014, the London borough of Newham established a primary-care based LTBI screening and treatment service in response to its high TB incidence: the 3-year average TB incidence was 100 per 100000 (20122014), higher than the average incidence in London (35.4 per 100000) [3]. More than half of the population in Newham (54\%) were born outside the UK and $86 \%$ of active TB cases in 2014 were notified among the foreign-born population $[12,13]$. The high TB rates in Newham and novel approach to TB control led to the programme being selected as a pilot for the national LTBI programme.

This study describes the screening outcomes of the LTBI testing and treatment pilot in Newham and determines factors contributing to LTBI screening uptake, IGRA test positivity and treatment uptake in order to inform LTBI screening programmes in England and other low-incidence countries.

\section{Methods}

Study design and cohort

We conducted a retrospective cohort study. We included all individuals identified as eligible for LTBI testing and treatment from 59 general practices (GPs) in the London borough of Newham between August 2014 and August 2015. Eligibility criteria included documented migrants who were born or who had spent $\geqslant 6$ months in a high-incidence country $(\geqslant 150$ per 100000 and sub-Saharan Africa) and had entered the UK within the last 5 years. Based on criteria for programmatic screening in the UK, individuals with pre-existing or previously treated LTBI or active TB were not eligible for testing and therefore not included in this study.

\section{Screening process}

The screening algorithm is outlined in figure 1. Eligible individuals were identified upon registering with a GP and offered LTBI testing. A single IGRA test was used to screen individuals. Individuals found to be IGRA positive were tested for HIV, hepatitis B and hepatitis C, and then reviewed by a GP to exclude active TB based on a history, physical examination and chest radiography. Individuals with signs or symptoms of suspected active TB (table 1), underlying liver disease or positive viral serology were referred to the local TB clinic in secondary care.

All other individuals diagnosed with LTBI (table 1) were prescribed 3 months of rifampicin and isoniazid combination therapy by the GP as recommended by national and international guidelines [14, 15]. Electronic prescriptions were sent to accredited community pharmacists who had been trained in providing LTBI treatment.

Support statement: M. Burman was funded by Bart's Charity and D. Zenner is affiliated with the National Institute for Health Research Health Protection Research Unit (NIHRHPRU) in Respiratory Infections at Imperial College London in partnership with Public Health England (PHE). The research received funding from the NIHR HPRU in Respiratory Infections at Imperial College London in Partnership with PHE. The views expressed are those of the authors and not necessarily those of the National Health Service, the NIHR, the Department of Health or PHE. Funding information for this article has been deposited with the Crossref Funder Registry. 


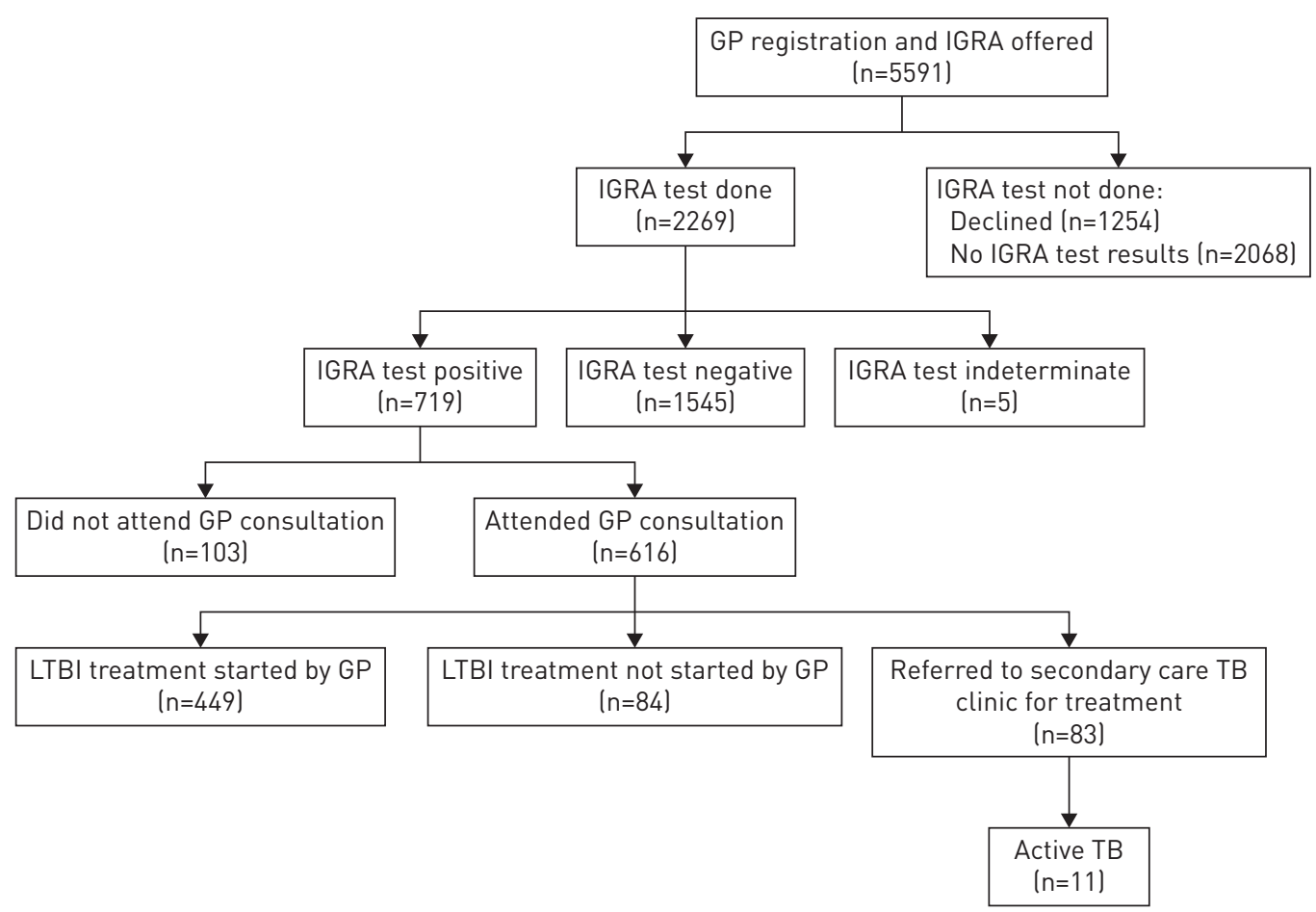

FIGURE 1 Latent tuberculosis infection (LTBI) testing and treatment programme flow chart. IGRA: interferon- $\gamma$ release assay; GP: general practitioner; TB: tuberculosis.

\section{Data collection and cleaning}

Data was collected using a bespoke template within the GP's electronic patient record system (EMISWeb; EMIS Health, Leeds, UK). Demographic information (age, sex, country of birth), co-morbidities (immunosuppression, diabetes mellitus, pre-existing lung, chronic liver disease and chronic kidney disease) and LTBI screening data (IGRA test result, HIV test result, hepatitis B and C serology results, electronic prescription for treatment and reason for referral to secondary care) were extracted from EMISWeb. The GP surgeries within Newham were categorised geographically into eight clusters responsible for the provision of care in that area, labelled Cluster 1-8.

Dates when LTBI testing was offered, the IGRA result was logged in the GP data system and the electronic prescription for treatment was issued were validated against each other and errors were excluded.

\section{Statistical analysis}

Logistic regression was performed for three steps along the patient pathway: LTBI testing uptake, IGRA positivity and LTBI treatment uptake (table 2). We report on LTBI testing uptake, not coverage (defined as those who accepted test among those who were eligible for screening). Although coverage is important from a population perspective, we estimate these figures from cumulative area returns, as we do not hold individual level data and do not present these here as not deemed robust enough. Analysis on treatment completion was beyond the scope of this paper, due to LTBI treatment being dispensed through pharmacists and therefore the data on treatment outcome was held in another database that is not

\section{TABLE 1 Definitions for LTBI and active TB cases}

\begin{tabular}{ll} 
Case term & Definition \\
\hline LTBI & IGRA test positivity and no clinical signs or symptoms suggestive of active TB. \\
Active TB & $\begin{array}{l}\text { Culture confirmed disease due to Mycobacterium tuberculosis complex or in the absence of } \\
\text { culture confirmation, a case with clinical signs and/or radiological signs and/or } \\
\text { symptoms compatible with active TB and the TB clinician decided to treat the individual } \\
\text { with a full course of anti-TB treatment. }\end{array}$
\end{tabular}

LTBI: latent tuberculosis infection; TB: tuberculosis; IGRA: interferon- $\gamma$ release assay. 


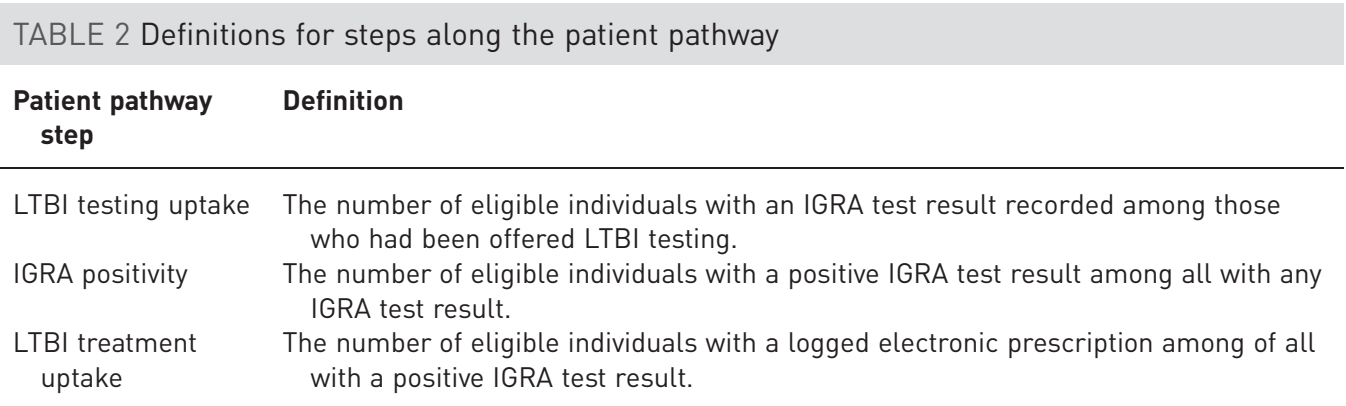

LTBI: latent tuberculosis infection; IGRA: interferon- $\gamma$ release assay.

currently linked to the primary care GP data. Demographic characteristics, co-morbidities and GP categories were used to compare eligible individuals at each step and odds ratios were calculated. Three forward stepwise multivariable logistic regression models were created, and a p-value of $<0.05$ was used to retain the variable in the model.

The time to take up LTBI testing was calculated as the time between the date that LTBI screening was offered and the date that the IGRA result was logged in the GP data system. Only individuals with an IGRA result were included in the LTBI testing delay analysis. LTBI testing delay was defined as more than 3 months between being offered LTBI testing and having the IGRA test done. The time to take up LTBI treatment was calculated using the time between the date that the IGRA result was logged and the date the electronic prescription for LTBI treatment was issued. Only those with an electronic prescription were included in the LTBI treatment delay analysis. LTBI treatment delay was defined as more than 1 month between having an IGRA test done and being prescribed LTBI treatment. Both LTBI testing and treatment delays were defined based on the distribution of the data and descriptively compared against demographic characteristics. Individuals missing required data to calculate delays were excluded from the analysis. There were no demographic differences between those with dates and those without. Demographic and GP factors were tested using univariable logistic regression to find associations with either LTBI testing delay or treatment delay.

Data were managed and analysed using Excel (Microsoft; Redmond, WA, USA) and STATA 13 (Statacorp; College Station, TX, USA).

\section{Results}

In total, 5591 individuals were offered LTBI testing between August 2014 and August 2015 in the London Borough of Newham. Most individuals were aged 16-35 years (3968; 71.0\%), were male $(3028 ; 54.2 \%)$ and born in the Indian sub-continent $(4387 ; 78.5 \%)$ (table 3 ). The five most common countries of birth of individuals offered LTBI testing were Bangladesh (1697; 30.3\%), India (1684; 30.1\%), Pakistan (851; 15.2\%), Nigeria (351; 6.3\%) and Somalia (189; 3.4\%) (Appendix 1 in the supplementary file). 539 (9.6\%) individuals offered LTBI testing had at least one co-morbidity and 840 (15.0\%) were current smokers (table 3).

\section{LTBI testing uptake}

Of those individuals offered LTBI testing, 2269 (40.6\%) had an IGRA test done, 1254 (22.4\%) declined LTBI testing and 2068 (37.0\%) had no IGRA result recorded (figure 1). There were no demographic differences between those who had declined LTBI testing and those who had been offered but had no IGRA result; therefore, all were considered as not having an IGRA test for the purpose of the analysis. Overall, LTBI testing uptake was highly variable month to month during the time period (between $38.1 \%$ and $60.4 \%$ ) and also varied by GP surgery with zero to $88.8 \%$ of individuals taking up LTBI testing.

The multivariable model for LTBI testing uptake, which adjusted for age, sex and all significant variables from univariable analysis, showed that individuals from East and South-East Asia and from sub-Saharan Africa were significantly less likely to take up LTBI testing compared with individuals from the Indian sub-continent (aOR 0.6 (95\% CI, 0.4-0.9) and aOR 0.7 (95\% CI, 0.6-0.8) respectively) (table 4). Individuals who currently smoked were less likely to take up LTBI testing (aOR 0.8 (95\% CI, 0.7-0.9)) and those with chronic liver disease were more likely to take up LTBI testing (aOR 1.6 (95\% CI, 1.1-2.5)). Diabetes mellitus was not significant in the multivariable model because age was a confounding factor, as $77.4 \%$ of diabetics were more than 36 years old. We compared the multivariable model with and without age and could validate the odds ratio for LTBI testing uptake among diabetics and, although it is strongly 
TABLE 3 Baseline characteristics of all individuals offered latent tuberculosis infection testing

n $(\%)$

\begin{tabular}{lc}
\hline Age group & \\
$<16$ years & $44(0.8)$ \\
$16-35$ years & $3968(71.0)$ \\
$36-50$ years & $1344(26.9)$ \\
$>50$ years & $235(1.4)$ \\
Sex & \\
Male & $3028(54.2)$ \\
Female & $2563(45.8)$ \\
Region of birth & \\
East and South-East Asia & $137(2.5)$ \\
Eastern Europe & $8(0.1)$ \\
Northern Africa & $11(0.2)$ \\
Southern Asia & $4387(78.5)$ \\
Sub-Saharan Africa & $1048(18.7)$ \\
Five most common countries of birth ${ }^{\#}$ & \\
Bangladesh & $1697(30.4)$ \\
India & $1684(30.1)$ \\
Pakistan & $851(15.2)$ \\
Nigeria & $351(6.3)$ \\
Somalia & $189(3.4)$ \\
Other & $819(14.6)$ \\
Co-morbidities & \\
Immunosuppression & $1(0.02)$ \\
Diabetes mellitus & $287(5.1)$ \\
Pre-existing lung disease & $182(3.3)$ \\
Chronic liver disease & $89(1.6)$ \\
Chronic kidney disease & $25(0.5)$ \\
Any comorbidity & $539(9.6)$ \\
Current smoker & $840(15.0)$ \\
\hline . See appendix 1 in the & \\
\hline
\end{tabular}

\#: See appendix 1 in the online supplementary material for all countries of birth listed.

influenced by age, diabetics are more likely to take up LTBI testing. The multivariable analysis was repeated excluding all individuals who were offered testing but had no IGRA result and the results were broadly similar.

The median (interquartile range) time to take up LTBI testing was 1.5 (0.4-3.4) months; almost half $(46.8 \%)$ of those who accepted LTBI testing had a delay of more than 1 month and $24.9 \%$ had a delay of more than 3 months. There were no significant differences in demographic characteristics between those that had a testing delay of less than 3 months and more than 3 months, and no difference in testing delay by IGRA result. The proportion of cases that experienced a testing delay varied by GP surgery from 0 to $100 \%$ experiencing a delay of more than 3 months. Among the eight GP surgery clusters, Cluster 3 had a significantly higher proportion of individuals who experienced a testing delay compared to Cluster 1, which offered the highest number of IGRA tests (OR 1.8 (95\% CI, 1.1-2.8)) (table 5).

\section{IGRA test positivity}

A total of $719(31.7 \%)$ individuals tested positive by IGRA, 1545 (68.1\%) had a negative result and five $(0.2 \%)$ had an indeterminate result (figure 1). IGRA positivity increased with age: $25.8 \%$ among $16-$ 35 year olds, $45.4 \%$ in $36-50$ year olds and $51.2 \%$ in those over 50 years old. The multivariable model showed that males, those aged over 36 years old, those born in sub-Saharan Africa and individuals with diabetes mellitus were significantly more likely to test positive after adjusting for sex, age and significant variables from the univariable analysis (aOR 1.4 (95\% CI, 1.2-1.7), aOR 2.1 (95\%CI, 1.7-2.6), aOR 2.5 (95\% CI, 1.7-3.7), aOR 1.6 (95\% CI, 1.3-2.1), aOR 1.6 (95\% CI, 1.1-2.3)) (table 6). Positive Bacillus Calmette-Guérin vaccination status was inversely correlated with IGRA positivity (OR 0.2 (95\% CI, 0.1-0.9)).

\section{LTBI treatment uptake}

Among individuals with a positive IGRA result, 616 (85.7\%) had attended a GP consultation to rule-out active TB (figure 1). Overall 83 individuals were referred to the TB clinic for specialist care because of HIV 
TABLE 4 Logistic regression model for latent tuberculosis infection (LTBI) testing uptake, comparing individuals with an interferon- $\gamma$ release assay (IGRA) test result (IGRA done) to individuals who were offered LTBI testing but had no recorded IGRA test result (IGRA not done)

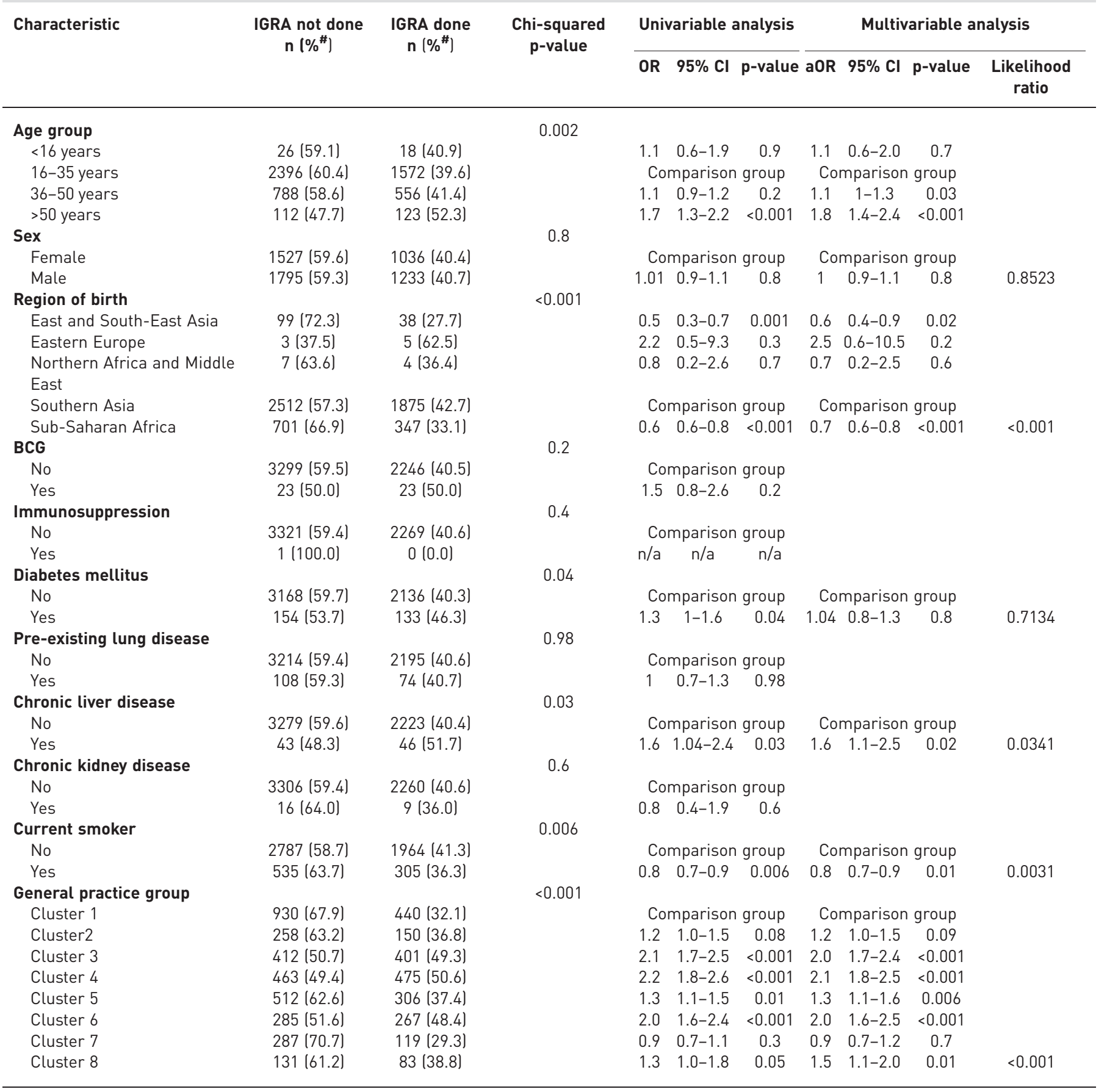

\#: proportions calculated out of each characteristic as row proportions.

(two individuals), hepatitis C (three) or hepatitis B (six) co-infection, or due to suspected active TB in individuals with at least one TB-related symptom (22) or an abnormal chest radiograph (five). The majority (40) of referred cases did not have a reason for referral recorded. Among those referred, a total of 11 active TB cases were notified, which accounts for a detection rate of 484.8 per 100000 individuals screened.

In total $449(62.5 \%)$ of those with a positive IGRA test took up LTBI treatment. LTBI treatment uptake varied between $37.5 \%$ in August 2014 and 58.5\% in July 2015; however, the increase was not significant 
TABLE 5 Latent tuberculosis infection (LTBI) screening and treatment referral uptake delays by general practice surgery cluster

\begin{tabular}{|c|c|c|c|c|c|c|c|c|c|c|c|c|}
\hline \multirow{2}{*}{$\begin{array}{c}\text { General } \\
\text { practice } \\
\text { cluster }\end{array}$} & \multirow{2}{*}{$\begin{array}{c}\text { General } \\
\text { practice } \\
\text { surgeries } \\
\text { n }\end{array}$} & \multirow{2}{*}{$\begin{array}{c}\text { Average } \\
\text { practice } \\
\text { list size } \\
n\end{array}$} & \multicolumn{5}{|c|}{$\begin{array}{c}\text { Delay between } \\
\text { LTBI test offered and IGRA test done }\end{array}$} & \multicolumn{5}{|c|}{$\begin{array}{l}\text { Delay between IGRA test done and } \\
\text { treatment referral }\end{array}$} \\
\hline & & & $\begin{array}{c}<3 \text { months } \\
\mathrm{n}\left(\%^{\#)}\right)\end{array}$ & $\begin{array}{c}>3 \text { months } \\
n\left(\%^{\#}\right)\end{array}$ & OR & $95 \% \mathrm{Cl}$ & $\overline{p \text {-value }}$ & $\begin{array}{c}<1 \text { month } \\
\text { n }\left(\%^{\#}\right)\end{array}$ & $\begin{array}{c}>1 \text { month } \\
n\left(\%^{\#}\right)\end{array}$ & $\mathrm{OR}$ & $95 \% \mathrm{Cl}$ & $p$-value \\
\hline Cluster 3 & 8 & 3618 & 167 (82.3) & 36 (17.7) & 0.6 & $0.4-1.0$ & 0.05 & $40(52.6)$ & $36(47.4)$ & 4.4 & $2.1-9.0$ & $<0.001$ \\
\hline Cluster 4 & 7 & 7002 & 75 (62.0) & $46(38.0)$ & 1.8 & $1.1-2.8$ & 0.02 & 40 (64.5) & 22 (35.5) & 2.7 & $1.2-5.7$ & 0.01 \\
\hline Cluster 5 & 11 & 6740 & 44 (67.7) & 21 (32.3) & 1.4 & $0.7-2.5$ & 0.3 & 29 (46.8) & 33 (53.2) & 5.5 & $2.6-11.7$ & $<0.001$ \\
\hline Cluster 6 & 6 & 7034 & 61 (69.3) & $27(30.7)$ & 1.3 & $0.7-2.2$ & 0.4 & $36(66.7)$ & 18 (33.3) & 2.4 & $1.1-5.4$ & 0.03 \\
\hline Cluster 7 & 6 & 6870 & $38(82.6)$ & $8(17.4)$ & 0.6 & $0.3-1.4$ & 0.2 & 28 (82.3) & $6(17.7)$ & 1 & $0.4-3.0$ & 0.9 \\
\hline Cluster 8 & 4 & 9858 & $45(90.0)$ & $5(10.0)$ & 0.3 & $0.1-0.8$ & 0.02 & 7 (87.5) & 1 (12.5) & 0.7 & $0.1-6.1$ & 0.7 \\
\hline
\end{tabular}

\#: Proportions calculated out of each general practice cluster as row proportions. IGRA: interferon- $\gamma$ release assay.

$(\mathrm{p}=0.1)$. No demographic or clinical factors were significantly associated with individuals being prescribed treatment over those that were not prescribed treatment (table 7). Although not significant, a higher proportion of men with an IGRA positive result were prescribed treatment, which may in part be due to pregnant women with a positive IGRA result not being prescribed treatment. Treatment uptake varied by GP surgery from 0 to $100 \%$ of LTBI cases taking up treatment. Accounting for demographic and clinical factors, GP surgery clusters 3 and 4 were significantly less likely to prescribe treatment compared with the GP cluster 1 ((aOR 0.6 (95\% CI, 0.3-0.9) and aOR 0.3 (95\% CI, 0.2-0.5)).

The median (interquartile range) time to take up treatment for individuals with a positive IGRA result was 17 (0-40) days. 138 (32.9\%) individuals had a LTBI treatment delay of more than 1 month. There were no demographic or clinical factors associated with treatment delay. However, the proportion of cases that experienced treatment delay varied by GP surgery from 0 to $100 \%$. Among the eight clusters of GP surgeries, four had a significantly higher proportion of LTBI cases who experienced a treatment delay of more than 1 month when compared with GP cluster 1 (table 5).

\section{Discussion}

This paper reports on the first year of a large community-based LTBI testing and treatment programme in the London borough of Newham. Overall screening uptake was low but we identified key demographic, clinical and provider factors associated with low uptake and therefore, lessons learned from Newham contain important policy implications for the implementation of LTBI testing and treatment in England and other low TB incidence countries.

Overall, less than half of the eligible population were tested for LTBI during the first year of the programme in Newham. A similarly large drop-off at this stage of the LTBI cascade of care was reported in a recent systematic review [16]. IGRA positivity was $32 \%$ in our study partly due to the inclusion of an older population in the first year of the pilot but also because the targeted population were screened based on country of birth. In this respect, our study was similar to other studies among migrant populations, where IGRA positivity varied between $25 \%$ and $30 \%$ and having LTBI was associated with increasing in-country TB incidence and age [17-20]. Overall, LTBI screening uptake and IGRA positivity were associated with demographic, clinical and provider related factors, whereas LTBI treatment uptake was exclusively provider-dependent and varied greatly by GP surgery. Our study also identified 11 active TB cases, which demonstrates the potential for LTBI screening programmes to have a larger impact on reducing further transmission of $\mathrm{TB}$, thus reducing significant morbidity and mortality.

We examined factors associated with LTBI testing uptake, which may allow for more targeted interventions to increase acceptance of the programme. Country of birth was an important influencing factor for both LTBI testing uptake and IGRA positivity because individuals from sub-Saharan Africa were less likely to take up testing whilst having a higher IGRA test positivity. This demonstrates the need for targeted interventions for the most at-risk patient populations. In the literature small-scale, setting-specific studies on LTBI screening have been described and systematic reviews on LTBI treatment uptake and completion have shown varied associations with migrant or refugee populations [21,22]. We are not aware 
TABLE 6 Logistic regression model for interferon- $\gamma$ release assay (IGRA) test positivity, comparing individuals with a positive IGRA test result to individuals with a negative IGRA test result

\begin{tabular}{|c|c|c|c|c|c|c|c|c|c|c|}
\hline \multirow[t]{2}{*}{ Characteristic } & \multirow{2}{*}{$\begin{array}{c}\text { IGRA negative } \\
\text { n }\left(\%^{\#}\right)\end{array}$} & \multirow{2}{*}{$\begin{array}{l}\text { IGRA positive } \\
\text { n }\left(\%^{\#}\right)\end{array}$} & \multirow{2}{*}{$\begin{array}{c}\text { Chi-squared } \\
\text { p value }\end{array}$} & \multicolumn{3}{|c|}{ Univariable analysis } & \multicolumn{4}{|c|}{ Multivariable analysis } \\
\hline & & & & OR & $95 \% \mathrm{Cl}$ & $\mathrm{p}$ value & $\mathrm{aOR}$ & $95 \% \mathrm{Cl}$ & $\mathrm{p}$ value & Likelihood ratio \\
\hline Age group & & & $<0.001$ & & & & & & & \\
\hline $16-35$ & $1166(74.2)$ & $406(25.8)$ & & \multicolumn{3}{|c|}{ Comparison group } & \multicolumn{3}{|c|}{ Comparison group } & \\
\hline $36-50$ & $301(54.6)$ & $250(45.4)$ & & 2.4 & $1.9-2.9$ & $<0.001$ & 2.1 & $1.7-2.6$ & $<0.001$ & \\
\hline$>50$ & $60(48.8)$ & 63 (51.2) & & 3.0 & $2.1-4.4$ & $<0.001$ & 2.5 & $1.7-3.7$ & $<0.001$ & \\
\hline Male & 797 (64.8) & $433(35.2)$ & & 1.4 & $1.2-1.7$ & $<0.001$ & 1.4 & $1.2-1.7$ & $<0.001$ & 0.0010 \\
\hline \multicolumn{11}{|l|}{ Region of birth } \\
\hline East and South East Asia & $27(71.1)$ & $11(28.9)$ & & 0.96 & $0.5-2.0$ & 0.9 & 0.8 & $0.4-1.7$ & 0.6 & \\
\hline Eastern Europe & $2(40.0)$ & $3(60.0)$ & & 3.5 & $0.6-21.2$ & 0.2 & 4.4 & $0.7-27.4$ & 0.1 & \\
\hline Northern Africa and Middle East & $3(75.0)$ & $1(25.0)$ & & 0.8 & $0.1-7.6$ & 0.8 & 0.6 & $0.06-6.4$ & 0.7 & \\
\hline Southern Asia & $1314(70.2)$ & $557(29.8)$ & & \multicolumn{3}{|c|}{ Comparison group } & \multicolumn{3}{|c|}{ Comparison group } & \\
\hline Diabetes mellitus & & & $<0.001$ & & & & & & & \\
\hline No & $1479(69.4)$ & $653(30.6)$ & & \multicolumn{3}{|c|}{ Comparison group } & \multicolumn{3}{|c|}{ Comparison group } & \\
\hline Yes & $66(50.0)$ & $66(50.0)$ & & 2.3 & $1.6-3.2$ & $<0.001$ & 1.6 & $1.1-2.3$ & 0.02 & 0.0240 \\
\hline Pre-existing lung disease & & & 0.4 & & & & & & & \\
\hline No & $1498(68.4)$ & $692(31.6)$ & & \multicolumn{3}{|c|}{ Comparison group } & \multicolumn{3}{|c|}{ Comparison group } & \\
\hline Yes & 47 (63.5) & 27 (36.5) & & 1.2 & $0.8-2.0$ & 0.4 & 1.04 & $0.6-1.7$ & 0.9 & 0.8406 \\
\hline Chronic liver disease & & & 0.003 & & & & & & & \\
\hline No & $1524(68.7)$ & 695 (31.3) & & \multicolumn{3}{|c|}{ Comparison group } & \multicolumn{3}{|c|}{ Comparison group } & \\
\hline Yes & $21(46.7)$ & 24 (53.3) & & 2.5 & $1.4-4.5$ & 0.002 & 1.8 & $1.0-3.4$ & 0.06 & 0.0728 \\
\hline Chronic kidney disease & & & 0.1 & & & & & & & \\
\hline No & $1541(68.3)$ & $714(31.7)$ & & \multicolumn{3}{|c|}{ Comparison group } & \multicolumn{3}{|c|}{ Comparison group } & \\
\hline Yes & $4(44.4)$ & $5(55.6)$ & & 2.7 & $0.7-10.1$ & 0.1 & 1.5 & $0.4-5.7$ & 0.6 & 0.5764 \\
\hline Current smoker & & & 0.8 & & & & & & & \\
\hline
\end{tabular}

\# : Proportions calculated out of each characteristic as row proportions.

of any other study which has examined additional demographics such as country of birth in detail, however other disease screening initiatives such as HPV vaccination [23] and HIV testing [24] have demonstrated similar differences among ethnic minorities.

Pre-existing health conditions may influence LTBI testing uptake. Individuals with diabetes mellitus or chronic liver disease were more likely to take up LTBI testing when offered. This may be because these individuals know they are at higher risk of progression from LTBI to active TB, and increased access to care through monitoring of their diabetes, which in turn influence health-seeking behaviour [25-27]. Populations with co-morbidities have been found in other studies to have higher LTBI treatment completion rates when compared with other populations, which could indicate greater concern about their own health or better access to care for persons with chronic conditions [16,22]. Older individuals were also more likely to take up LTBI testing and had higher IGRA positivity which may be confounded by older individuals having more co-morbidities, but may also be influenced by similar health-seeking behaviour. Conversely, other studies have shown that current smokers or individuals with social risk factors, such as homelessness, injecting drug use and/or alcohol use are less likely to adhere to LTBI treatment, which supports our finding that current smokers were less likely to take up LTBI testing and suggests that health-seeking behaviours and access to care play an important role in LTBI testing and treatment programmes $[21,22,28]$.

We tested all demographic and clinical factors and found no correlation with treatment uptake; however, the same model showed that treatment uptake varied widely by GP surgery and several geographic GP clusters were more likely to prescribe LTBI treatment than others. This association with providers can be 
TABLE 7 Logistic regression model for latent tuberculosis infection (LTBI) treatment prescription uptake, comparing individuals with a positive interferon $\gamma$ release assay (IGRA) test result who had an electronic prescription for LTBI treatment to those who did not have an electronic prescription

\begin{tabular}{|c|c|c|c|c|c|c|c|c|c|}
\hline \multirow[t]{2}{*}{ Characteristic } & \multirow{2}{*}{$\begin{array}{c}\text { No treatment } \\
\text { prescribed } \\
n\left(\%^{\#)}\right.\end{array}$} & \multirow{2}{*}{$\begin{array}{c}\text { Treatment } \\
\text { prescribed } \\
\mathrm{n}\left(\%^{\#}\right)\end{array}$} & \multirow[t]{2}{*}{$\begin{array}{c}\text { Chi-squared } \\
\text { p value }\end{array}$} & \multicolumn{3}{|c|}{$\begin{array}{c}\text { Univariable } \\
\text { analysis }\end{array}$} & \multicolumn{3}{|c|}{$\begin{array}{l}\text { Multivariable } \\
\text { analysis }\end{array}$} \\
\hline & & & & OR & $95 \% \mathrm{Cl}$ & $p$ value & OR & $95 \% \mathrm{Cl}$ & $\mathrm{p}$ value \\
\hline$<16$ & $0(0)$ & $0(0)$ & & & & & & & \\
\hline $16-35$ & $115(38.2)$ & $251(61.8)$ & & \multicolumn{3}{|c|}{ Comparison group } & \multicolumn{3}{|c|}{ Comparison group } \\
\hline $36-50$ & $89(35.6)$ & $161(64.4)$ & & 1.1 & $0.8-1.5$ & 0.5 & 1.0 & $0.7-1.5$ & 0.8 \\
\hline female & $119(41.6)$ & $167(58.4)$ & & \multicolumn{3}{|c|}{ Comparison group } & \multicolumn{3}{|c|}{ Comparison group } \\
\hline male & $151(34.9)$ & $282(65.1)$ & & 1.3 & $0.9-1.8$ & 0.07 & 1.4 & $1.0-2.0$ & 0.06 \\
\hline Region of birth & & & 0.7 & & & & & & \\
\hline East and South East Asia & $5(45.5)$ & $6(54.5)$ & & 0.7 & $0.2-2.3$ & 0.6 & 0.5 & $0.1-1.7$ & 0.3 \\
\hline Eastern Europe & $2(66.7)$ & $1(33.3)$ & & 0.3 & $0.03-3.3$ & 0.3 & 0.3 & $0.02-3.2$ & 0.3 \\
\hline $\begin{array}{l}\text { Northern Africa and Middle } \\
\text { East }\end{array}$ & $0(0.0)$ & $1(100.0)$ & & - & - & - & - & - & - \\
\hline no & $269(37.5)$ & 448 (62.5) & & \multicolumn{3}{|c|}{ Comparison group } & \multicolumn{3}{|c|}{ Comparison group } \\
\hline yes & $1(50.0)$ & $1(50.0)$ & & 0.6 & $0.04-9.6$ & 0.70 & 0.3 & $0.02-5.7$ & 0.4 \\
\hline Diabetes mellitus & & & 0.4 & & & & & & \\
\hline no & $242(37.1)$ & $411(62.9)$ & & \multicolumn{3}{|c|}{ Comparison group } & \multicolumn{3}{|c|}{ Comparison group } \\
\hline yes & $28(42.4)$ & $38(57.6)$ & & 0.8 & $0.5-1.3$ & 0.4 & 0.8 & $0.4-1.3$ & 0.4 \\
\hline Pre-existing lung disease & & & 0.9 & & & & & & \\
\hline no & $260(37.6)$ & $432(62.4)$ & & \multicolumn{3}{|c|}{ Comparison group } & \multicolumn{3}{|c|}{ Comparison group } \\
\hline yes & $10(37.0)$ & $17(63.0)$ & & 1.0 & $0.5-2.3$ & 0.9 & 1.0 & $0.4-2.2$ & 0.9 \\
\hline Chronic liver disease & & & 0.2 & & & & & & \\
\hline no & $258(37.1)$ & $437(62.9)$ & & \multicolumn{3}{|c|}{ Comparison group } & \multicolumn{3}{|c|}{ Comparison group } \\
\hline yes & $12(50.0)$ & $12(50.0)$ & & 0.6 & $0.3-1.3$ & 0.2 & 0.5 & $0.2-1.3$ & 0.2 \\
\hline Chronic kidney disease & & & 0.9 & & & & & & \\
\hline Cluster 4 & $82(55.0)$ & $67(45.0)$ & & 0.3 & $0.2-0.5$ & $<0.001$ & 0.3 & $0.2-0.5$ & $<0.001$ \\
\hline Cluster 5 & $32(34.0)$ & $62(66.0)$ & & 0.8 & $0.4-1.3$ & 0.3 & 0.7 & $0.4-1.2$ & 0.2 \\
\hline Cluster 6 & 25 (31.7) & 54 (68.3) & & 0.8 & $0.4-1.5$ & 0.6 & 0.8 & $0.4-1.4$ & 0.4 \\
\hline Cluster 7 & $18(31.6)$ & 39 (68.4) & & 0.8 & $0.4-1.6$ & 0.6 & 0.9 & $0.4-1.8$ & 0.8 \\
\hline Cluster 8 & 5 (33.3) & $10(66.7)$ & & 0.8 & $0.2-2.4$ & 0.7 & 0.8 & $0.2-2.6$ & 0.7 \\
\hline
\end{tabular}

\#: Proportions calculated out of each characteristic as row proportions.

explained by the differences in treatment delay, as GP surgeries that had longer treatment delays also had patients who were less likely to take up treatment. LTBI testing and treatment programmes in primary care can be complex and require training and incentives for GPs. A recent survey of GPs in England to find the enablers and barriers to testing and treating LTBI in primary care also confirmed the importance of training health care staff to feel confident in their work [29]. Training is provided to all GPs involved in the Newham LTBI screening programme and is mandatory for them to attend in order to stay in the programme; however, it is possible that implementation of the programme may differ based on available resources to offer screening, availability of appointments to offer treatment, knowledge, skills and beliefs. This study demonstrates the importance of identifying practices and clusters of practices with poorer treatment uptake in order to more closely engage them in training and support activities. 
A higher proportion of men were prescribed LTBI treatment compared with women, which is most likely due to the fact that pregnant women were not offered treatment until post-partum, as specified by the early screening rules of the pilot site. Early results from this study demonstrating the risk of lower uptake of women in childbearing age informed a revision of the pathway to allow for screening of pregnant women and a call-recall system for treatment after pregnancy.

This study has several limitations. We present observational data, where data collection was not mandatory. This could have led to missing data and potential loss of statistical power for some analysis. We analysed and compared the baseline characteristics of those with complete records and with missing data and found these were broadly comparable; we think it likely that data were missing at random, not introducing bias. There may have also been coding errors used for categorising the co-morbidities; however, we do not think this has an effect in the analysis because there were no systematic errors found in the data. As this was an observational study, it is possible, that not all IGRA test results were recorded, which could have decreased the LTBI testing uptake rate and could have resulted in an overestimation of IGRA positivity if negative results were not recorded. However, we have no evidence for systematic under-recording by demographic or provider characteristics. Another limitation was that we were not able to report on LTBI screening coverage or on treatment outcomes, which were out of scope for this paper; however, the absence of these indicators does not affect the validity of our findings from the screening pathway.

In conclusion, our evaluation of the LTBI testing and treatment project in Newham illustrates the feasibility and acceptability of primary-care based LTBI screening and treatment programmes. Our study shows that particular migrant population groups require more patient engagement to ensure high testing and treatment uptake particularly if uptake is low and/or LTBI positivity is high. This study also showed the immediate impact that LTBI screening programmes have on TB control by identifying active TB cases earlier, reducing further transmission. The testing and treatment delays found in this pilot were high and possibly adversely impacted on uptake. The innovative approach to LTBI screening through primary-care registrations has many positive implications on increasing accessibility within most migrant communities, however, special programmes are needed to meet the needs of undocumented migrants, asylum seekers, refugees and other underserved populations who may not engage with health services and therefore are missed by this programme. Whilst this paper identifies important factors related to LTBI testing and treatment uptake, larger studies are urgently needed to better understand the barriers and enablers to this intervention in order for it to effectively contribute to TB elimination worldwide.

\section{Acknowledgments}

The authors would like to acknowledge the support of the National Institute for Health Research Health Protection Research Unit (NIHR HPRU) in Respiratory Infections at Imperial College London. Also we would like to thank the Clinical Effectiveness Group at Queen Mary University for providing the data output from EMIS.

Author contributions: This study was initially conceived by M.G. Loutet, D. Zenner, H. Kunst and M. Burman, all of whom made substantial contributions to the paper. Data analysis and first draft of paper was done by M.G. Loutet. D. Zenner was the supervisor for the paper and guided and informed analysis and writing. All authors contributed comments on analysis and interpretation, and approved the final draft.

\section{References}

1 WHO Global TB Programme. Factsheet. The End TB Strategy. Global strategy and targets for tuberculosis prevention, care and control after 2015. Available from www.who.int/tb/post2015_TBstrategy.pdf Date last accessed: July 15, 2017.

2 Getahun H, Matteelli A, Abubakar I, et al. Management of latent Mycobacterium tuberculosis infection: WHO guidelines for low tuberculosis burden countries. Eur Respir J 2015; 46: 1563-1576.

3 Public Health England. TB in England Annual Report 2015 (presenting data on 2014). Available from www.gov. uk/government/uploads/system/uploads/attachment_data/file/564649/TB_annual_report_2015.pdf Date last accessed: July 15, 2017; Date last updated: January 2016.

4 Hamblion EL, Le Menach A, Anderson LF, et al. Recent TB transmission, clustering and predictors of large clusters in London, 2010-2012: results from first 3 years of universal MIRU-VNTR strain typing. Thorax 2016; 71: 749-756.

5 Ricks PM, Cain KP, Oeltmann JE, et al. Estimating the burden of tuberculosis among foreign-born persons acquired prior to entering the U.S., 2005-2009. PLOS ONE 2011; 6: e27405.

6 Sharma SK, Sharma A, Kadhiravan T, et al. Rifamycins (rifampicin, rifabutin and rifapentine) compared to isoniazid for preventing tuberculosis in HIV-negative people at risk of active TB. Cochrane Database Syst Reviews 2013; 7: CD007545.

7 Stagg HR, Zenner D, Harris RJ, et al. Treatment of latent tuberculosis infection A network meta-analysis Treatment of latent tuberculosis infection. Ann Intern Med 2014; 161: 419-428.

8 Pareek M, Watson JP, Ormerod LP, et al. Screening of immigrants in the UK for imported latent tuberculosis: a multicentre cohort study and cost-effectiveness analysis. Lancet Infect Dis 2011; 11: 435-444.

9 Panchal RK, Browne I, Monk P, et al. The effectiveness of primary care based risk stratification for targeted latent tuberculosis infection screening in recent immigrants to the UK: a retrospective cohort study. Thorax 2014; 69: 354-362. 
10 Zammarchi L, Casadei G, Strohmeyer M, et al. A scoping review of cost-effectiveness of screening and treatment for latent tuberculosis infection in migrants from high-incidence countries. BMC Health Serv Res 2015; 15: 412.

11 Public Health England N-E. Collaborative TB Strategy for England 2015-2020. Available from www.gov.uk/ government/uploads/system/uploads/attachment_data/file/403231/Collaborative_TB_Strategy_for_England_2015_ 2020_.pdf Date last accessed: July 15 2017; date last updated: January 2015.

12 Office for National Statistics. Population of the UK by Country of Birth and Nationality: 2015. Office for National Statistics. Available from www.ons.gov.uk/peoplepopulationandcommunity/populationandmigration/ internationalmigration/datasets/populationoftheunitedkingdombycountryofbirthandnationality Date last accessed: July 152017.

13 Public Health England. Tuberculosis in London: Annual review (2014 data). Public Health England. Available from www.gov.uk/government/uploads/system/uploads/attachment_data/file/484927/Annual_review_of_ tuberculosis_in_London_2014_data.pdf Date last accessed: July 15, 2017; date last updated: December 2014.

14 NICE. Tuberculosis NICE guidelines. Available from www.nice.org.uk/guidance/ng33/chapter/recommendations\# latent-tb Date last accessed: September 22, 2017; date last updated: May 2016

15 WHO. Guidelines on the management of latent tuberculosis infection. Available from www.who.int/tb/ publications/ltbi_document_page/en/ Date last accessed: September 21, 2017; date last updated: 2015.

16 Alsdurf H, Hill PC, Matteelli A, et al. The cascade of care in diagnosis and treatment of latent tuberculosis infection: a systematic review and meta-analysis. Lancet Infect Dis 2016; 16: 1269-1278.

17 Chen $\mathrm{C}$, Zhu T, Wang Z, et al. High latent TB infection rate and associated risk factors in the eastern China of low TB incidence PLoS ONE 2015; 10: e0141511.

18 Pareek M, Greenaway C, Noori T, et al. The impact of migration on tuberculosis epidemiology and control in high-income countries: a review. BMC Med 2016; 14: 48.

19 Pareek M, Bond M, Shorey J, et al. Community-based evaluation of immigrant tuberculosis screening using interferon $\gamma$ release assays and tuberculin skin testing: observational study and economic analysis. Thorax 2013; 68: $230-239$.

20 Rennert-May E, Hansen E, Zadeh T, et al. A Step toward tuberculosis elimination in a low-incidence country: successful diagnosis and treatment of latent tuberculosis infection in a refugee clinic. Can Respir J 2016; 2016: 7980869.

21 Stuurman AL, Vonk Noordegraaf-Schouten M, van Kessel F, et al. Interventions for improving adherence to treatment for latent tuberculosis infection: a systematic review. BMC Infect Dis 2016; 16: 257.

22 Sandgren A, Vonk Noordegraaf-Schouten M, van Kessel F, et al. Initiation and completion rates for latent tuberculosis infection treatment: a systematic review. BMC Infect Dis 2016; 16: 204.

23 Fernández de Casadevante V, Gil Cuesta J, Cantarero-Arévalo L. Determinants in the uptake of the human papillomavirus vaccine: a systematic review based on european studies. Front Oncol 2015; 5: 141.

24 Álvarez-del Arco D, Monge S, Rivero-Montesdeoca Y, et al. Implementing and expanding HIV testing in immigrant populations in Europe: Comparing guideline's recommendations and expert's opinions. Enferm Infecc Microbiol Clin 2017; 35: 47-51.

25 Jeon CY, Murray MB. Diabetes Mellitus increases the risk of active tuberculosis: a systematic review of 13 observational studies. PLoS Med 2008; 5: e152.

26 Ai J-W, Ruan Q-L, Liu Q-H, et al. Updates on the risk factors for latent tuberculosis reactivation and their managements. Emerg Microbes Infect 2016; 5: e10.

27 Lönnroth K, Roglic G, Harries AD. Improving tuberculosis prevention and care through addressing the global diabetes epidemic: from evidence to policy and practice. Lancet Diabetes Endocrinol 2014; 2: 730-739.

28 Lavigne M, Rocher I, Steensma C, et al. The impact of smoking on adherence to treatment for latent tuberculosis infection. BMC Public Health 2006; 6: 66.

29 Atchison C, Zenner D, Barnett L, et al. Treating latent TB in primary care: a survey of enablers and barriers among UK General Practitioners. BMC Infect Dis 2015; 15: 331. 